Research Paper

\title{
Metachronous Brain Metastasis in patients with EGFR-mutant NSCLC indicates a worse prognosis
}

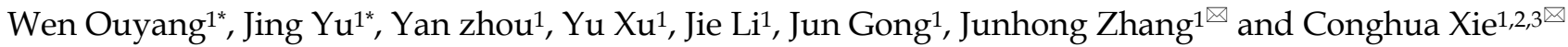 \\ 1. Department of Radiation and Medical Oncology, Zhongnan Hospital, Wuhan University, Wuhan, China. \\ 2. Hubei Key Laboratory of Tumor Biological Behaviors, Zhongnan Hospital, Wuhan University, Wuhan, China. \\ 3. Hubei Clinical Cancer Study Center, Zhongnan Hospital of Wuhan University, Wuhan, China. \\ *Both authors contributed equally as co-first authors. \\ $\triangle$ Corresponding authors: Conghua Xie and Junhong Zhang, Department of Radiation and Medical Oncology, Zhongnan Hospital of Wuhan University, 169 \\ Donghu Road, Wuchang District, Wuhan, Hubei 430071, China. E-mail: chxie_65@whu.edu.cn (CX); and zjhzhongnan@163.com (JZ). Phone: 86-27-67812607;
} Fax: 86-27-67812889.

(c) The author(s). This is an open access article distributed under the terms of the Creative Commons Attribution License (https://creativecommons.org/licenses/by/4.0/). See http://ivyspring.com/terms for full terms and conditions.

Received: 2020.03.29; Accepted: 2020.09.23; Published: 2020.10.21

\begin{abstract}
Purpose: NSCLC patients with EGFR mutation were associated with high incidence of brain metastasis (BM). BM could be grouped by the time of occurrence, including synchronous BM at initial diagnosis and metachronous BM during disease course. The primary aim of the study was to investigate the survival of patients with metachronous BM.

Methods: A total of 99 EGFR-mutant advanced NSCLC patients in our institute between 2012 and 2018 were grouped into synchronous BM and metachronous BM. Comparisons of OS were performed based on BM status. The independent prognostic factors of OS were investigated, and extracranial and intracranial PFS were further analyzed.

Results: Patients with metachronous BM (mOS: 22.1 months) had poorer outcomes than synchronous BM (mOS: 30.3 months) $(P=0.016)$. Moreover, multivariate analysis indicated that $B M$ status $(P=0.015)$, local therapy for $B M(P=0.013)$ and subsequent treatment of Osimertinib $(P=0.008)$ impact significantly on OS. Significantly, the proportion of local therapy for BM had no difference between patients with synchronous and metachronous BM. And patients with metachronous BM harbored a more favorable prognostic factor (higher proportion of subsequent Osimertinib treatment), but also harbored a poorer prognostic factor (metachronous BM), which confirmed BM status was the most significant prognostic factor of OS. At last, results of extracranial and intracranial PFS indicated that patients with metachronous BM tended to have a higher risk of intracranial disease progression.

Conclusions: Patients developing metachronous BM during EGFR-TKIs treatment have worse outcomes than synchronous BM. Our findings suggested that the patients with metachronous BM should receive more aggressive treatments.
\end{abstract}

Key words: non-small cell lung cancer, epidermal growth factor receptor, brain metastases, synchronous, metachronous

\section{Introduction}

Non-small cell lung cancer (NSCLC) is one of the malignancies with the highest morbidity and mortality [1]. Brain is a frequent site of NSCLC metastasis, and patients with brain metastasis (BM) are associated with high mortality and poor life quality [2]. The median survival in patients who suffer BM without treatment is less than 1 month, and the median survival after whole brain radiation therapy (WBRT) is only 3-6 months [3]. Additionally, $\mathrm{BM}$ could be grouped by the time of occurrence, including synchronous $\mathrm{BM}$ and metachronous $\mathrm{BM}$. Approximately $10 \%$ of NSCLC patients are synchronous BM at their initial diagnosis, and $40-50 \%$ of patients develop metachronous BM during the course of the disease [4]. 
Epidermal growth factor receptor (EGFR) mutation is a favorable prognostic marker of NSCLC. EGFR-tyrosine kinase inhibitors (TKIs) largely improved the survival of EGFR-mutant advanced NSCLC patients [5-7]. However, patients with EGFR mutation were associated with higher incidence of developing BM compared to patients with wild type EGFR [8-10]. Moreover, the longer survival of patients with EGFR mutant NSCLC is associated with higher exposure risk to $\mathrm{BM}$ [8]. Therefore, patients with EGFR mutant NSCLC tend to have a high risk of developing metachronous BM. Phase III trials of EGFR-TKIs for EGFR-mutant advanced NSCLC reported patients with $\mathrm{BM}$ had a worse median overall survival (OS) than the patients without BM, but synchronous BM and metachronous BM are seldom differentiated in these trials [11-13]. There is a lack of sufficient data on the prognosis of metachronous BM among EGFR-mutant advanced NSCLC patients. Therefore, it was necessary to evaluate the prognosis of EGFR-mutant advanced NSCLC patients with metachronous BM.

Consequently, we established a retrospective single-institutional database including consecutive EGFR-mutant advanced NSCLC patients with synchronous BM or metachronous BM from January 2012 and June 2018, to evaluate the survival of patients grouped by $\mathrm{BM}$ status, to explore the prognostic factors of OS, and to analyze potential mechanism of different outcomes based on BM status.

\section{Methods}

\section{Patients}

Between January 2012 and June 2018, a total of 229 consecutive patients with EGFR-mutant advanced NSCLC were included at the Department of Radiation and Medical Oncology, Zhongnan Hospital of Wuhan University. Among them, three patients were excluded due to short EGFR-TKI treatment $(<1$ month), and 127 patients without BM were excluded. A total of 99 eligible patients with synchronous or metachronous BM were enrolled in this study. Our inclusion criteria are: (1) NSCLC was confirmed by cytology (8 pts), or histology (91 pts) (World Health Organization, WHO); (2) EGFR mutations were confirmed by real-time quantitative PCR (ARMS, 83 pts) or Next Generation Sequencing (NGS, 16 pts), using histological or cytological specimens from primary or metastatic lesions; (3) The disease was clinically diagnosed as stage IIIB (2 pts)-IV (97 pts) (American Joint Committee on Cancer, the 7th Edition); (4) The patients were treatment naive for EGFR-TKIs treatment; (5) All patients received brain Magnetic Resonance Imaging (MRI) scans within 1 month before EGFR-TKIs treatment. A total of 69 patients were synchronous BM at initial diagnosis, and 30 patients developed metachronous BM during EGFR-TKIs treatment. The clinical and therapeutic characteristics of these included patients grouped by BM status are shown in Table 1.

\section{Treatment and follow up}

Among the 99 patients with synchronous or metachronous $\mathrm{BM}, 16$ patients received chemotherapy as their first-line therapy, and the other 83 patients received EGFR-TKIs treatment initially. EGFR-TKIs (gefitinib, erlotinib, or icotinib) were continuously administered until progression of disease (PD) or intolerable side effects. Treatment beyond PD was allowed on the judgement of continuously clinical benefit by the oncologists.

Follow-up examinations were performed every 2 months, including thoracic and abdominal CT scans, brain MRI scans. Progression-free survival (PFS) was defined as the time from EGFR-TKIs treatment to PD (including local, regional, or distant progression) or death from any cause. OS was defined as the time from EGFR-TKIs treatment to death from any cause. Extracranial PFS was defined as the time from EGFR-TKIs treatment to extracranial PD (excluding intracranial PD) or death from any cause. Intracranial PFS (iPFS) was defined as the time from EGFR-TKIs treatment to intracranial PD (including appearance of new intracranial lesions, or existing intracranial lesions progression) or death from any cause. Intracranial PFS after brain radiotherapy (BRT) was defined as the time from radiotherapy for intracranial lesions (71 patients who received BRT) or from EGFR-TKIs treatment (28 patients who didn't receive BRT) to intracranial PD or death from any cause. Treatment responses were evaluated by the response evaluation criteria in solid tumors as complete response $(\mathrm{CR})$, partial response $(\mathrm{PR})$, stable $(\mathrm{SD})$, and progression (PD).

\section{Statistics}

All statistical analyses were conducted using Statistical Package for Social Scientists (SPSS/ Windows, Version 22.0, SPSS Inc., Chicago, USA). Descriptive statistics were used for categorical variables (frequency and percentage) and continuous variables (median and range). The cumulative incidence of survival was calculated by the Kaplan-Meier method with 95\% confidence intervals (CIs). Univariable and multivariable Cox regression analyses were performed to explore the prognostic factors associated with OS. The multivariable Cox regression analysis simultaneously included those factors that had shown associations $(P<0.100)$ in the 
univariable Cox regression analyses. All tests were two-sided and $P<0.05$ were considered statistically significant.

Table 1. Baseline and treatment characteristics of patients grouped by BM status

\begin{tabular}{|c|c|c|c|c|}
\hline \multirow[t]{2}{*}{ Characteristic } & \multicolumn{2}{|c|}{$\begin{array}{l}\text { Patients developing } \\
\text { metachronous BM } \\
(\mathrm{n}=30)\end{array}$} & \multicolumn{2}{|c|}{$\begin{array}{l}\text { Patients with } \\
\text { synchronous BM } \\
(\mathrm{n}=69)\end{array}$} \\
\hline & NO. & $\%$ & NO. & $\%$ \\
\hline \multicolumn{5}{|l|}{ Age, years } \\
\hline Median (Range) & \multicolumn{2}{|c|}{$54(33-75)$} & \multicolumn{2}{|c|}{$54(36-73)$} \\
\hline \multicolumn{5}{|l|}{ Gender } \\
\hline Male & 14 & 46.7 & 22 & 31.9 \\
\hline Female & 16 & 53.3 & 47 & 68.1 \\
\hline \multicolumn{5}{|l|}{ KPS score } \\
\hline$\geq 80$ & 30 & 100 & 58 & 84.1 \\
\hline$<80$ & 0 & 0 & 11 & 15.9 \\
\hline \multicolumn{5}{|l|}{ Histology } \\
\hline Adenocarcinoma & 28 & 93.3 & 66 & 95.7 \\
\hline Non-adenocarcinoma & 2 & 6.7 & 3 & 4.3 \\
\hline \multicolumn{5}{|l|}{ BMI } \\
\hline Mean $(95 \% \mathrm{CI})$ & \multicolumn{2}{|c|}{$22.7(16.3-29.2)$} & \multicolumn{2}{|c|}{$22.0(16.3-27.7)$} \\
\hline \multicolumn{5}{|l|}{ Smoking status } \\
\hline Yes & 8 & 26.7 & 12 & 17.4 \\
\hline No & 22 & 73.3 & 57 & 82.6 \\
\hline \multicolumn{5}{|l|}{ CEA (ng/ml) } \\
\hline Median (Range) & \multicolumn{2}{|c|}{$30.5(1.5-1819)$} & \multicolumn{2}{|c|}{$21.5(0.6-2278)$} \\
\hline \multicolumn{5}{|l|}{ CA125 (ng/ml) } \\
\hline Median (Range) & \multicolumn{2}{|c|}{$69.4(11.3-954.5)$} & 37.0 & $5-5304)$ \\
\hline $\mathrm{NSE}(\mathrm{ng} / \mathrm{ml})$ & & & & \\
\hline Median (Range) & 15.2( & $-55.2)$ & 13.8 & $.7-70.9)$ \\
\hline First-line treatment regimen & & & & \\
\hline EGFR-TKI treatment & 21 & 70 & 62 & 89.9 \\
\hline Chemotherapy & 9 & 30 & 7 & 10.1 \\
\hline Type of EGFR mutations & & & & \\
\hline Exon 21 point & 14 & 46.7 & 25 & 36.2 \\
\hline Exon 19 deletion & 11 & 36.7 & 36 & 52.2 \\
\hline Other & 5 & 16.7 & 8 & 11.6 \\
\hline NO. of extracranial metastases & & & & \\
\hline 0 & 2 & 6.7 & 0 & 0.0 \\
\hline 1 & 14 & 46.7 & 26 & 37.7 \\
\hline 2 & 10 & 33.3 & 23 & 33.3 \\
\hline 3 or more & 4 & 13.3 & 20 & 29.0 \\
\hline Clinical stages & & & & \\
\hline Stage IIIB & 2 & 6.7 & 0 & 0.0 \\
\hline Stage IV & 28 & 93.3 & 69 & 100 \\
\hline Location of extracranial metastatic sit & & & & \\
\hline Pleural effusion & 6 & 20.0 & 2 & 2.9 \\
\hline Liver & 4 & 13.3 & 6 & 8.7 \\
\hline Adrenal & 1 & 3.3 & 9 & 13.0 \\
\hline Bone & 18 & 60 & 27 & 39.1 \\
\hline Lung & 17 & 56.6 & 25 & 36.2 \\
\hline Other & 2 & 6.7 & 7 & 10.1 \\
\hline Type of EGFR-TKIs & & & & \\
\hline Gefitinib & 19 & 63.3 & 44 & 63.8 \\
\hline Erlotinib & 7 & 23.3 & 19 & 27.5 \\
\hline Icotinib & 4 & 13.4 & 6 & 8.7 \\
\hline Symptomatic BM & 9 & 30.0 & 34 & 49.3 \\
\hline Local therapy for BM & & & & \\
\hline None & 8 & 26.7 & 20 & 29.0 \\
\hline WBRT & 14 & 46.7 & 30 & 43.5 \\
\hline SRS & 8 & 26.6 & 19 & 27.5 \\
\hline Radiotherapy for primary lesion & 10 & 33.3 & 19 & 27.5 \\
\hline Subsequent treatment of Osimertinib & 13 & 43.3 & 16 & 23.2 \\
\hline Diagnosed as oligometastasis & 7 & 23.3 & 17 & 24.6 \\
\hline
\end{tabular}

\section{Results}

\section{Patient characteristics}

A total of 99 eligible patients with BM were analyzed in the retrospective study. All patients received brain MRI scans before EGFR-TKIs treatment. Among them, 69 patients were confirmed as synchronous BM at initial diagnosis, the other 30 patients showed negative results of brain MRI scans before EGFR-TKIs treatment, whereas developed metachronous BM during EGFR-TKIs treatment. The clinical and therapeutic characteristics of these patients grouped by BM status are shown in Table $\mathbf{1}$. The median age of the patients that would develop metachronous BM and patients with synchronous BM were both 54 years. There was no difference between the two groups with respect to gender, histology, BMI, smoking status, tumor markers levels, clinical stages, extracranial metastases, type of EGFR mutations, type of EGFR-TKIs, diagnosed as oligometastasis or not, and whether local therapy for $\mathrm{BM}$ or primary lesion. However, due to our study is a limited-sample retrospective study, the distribution of KPS score and subsequent treatment of Osimertinib between the two groups was imbalanced (KPS score $\geq$ 80: $100 \%$ patients that would develop metachronous BM vs. $84.1 \%$ patients with synchronous BM; and subsequent treatment of Osimertinib: $43.3 \%$ patients that would develop metachronous BM vs. $23.2 \%$ patients with synchronous BM). Additionally, the proportion of symptomatic BM in patients with metachronous BM (30\%) was lower than synchronous BM (49.3\%).

\section{Overall survival of patients grouped by BM status}

For 99 EGFR-mutant advanced NSCLC patients with $\mathrm{BM}$, the median duration of follow-up was 22.8 months (95\% CI: 17.5-28.1 months). The median OS of these patients was 29.3 months (95\% CI: 19.5-39.1 months). The 1-, 2- and 3-year OS rates were $91.5 \%$, $55.4 \%$ and $33.4 \%$ respectively.

To evaluate the impact of BM status on OS, these 99 patients were grouped by synchronous BM and metachronous BM. Compared with patients with synchronous $\mathrm{BM}$, patients developing metachronous $\mathrm{BM}$ during the course of EGFR-TKIs treatment were at a higher risk on $\mathrm{OS}(\mathrm{HR}=2.17,95 \% \mathrm{CI}$ : 1.14-4.12; median OS: 22.1 months of patients with metachronous BM vs. 30.3 months of patients with synchronous BM, Figure 1). Our findings indicated that metachronous $\mathrm{BM}$ was a significantly poor prognostic factor for OS. 


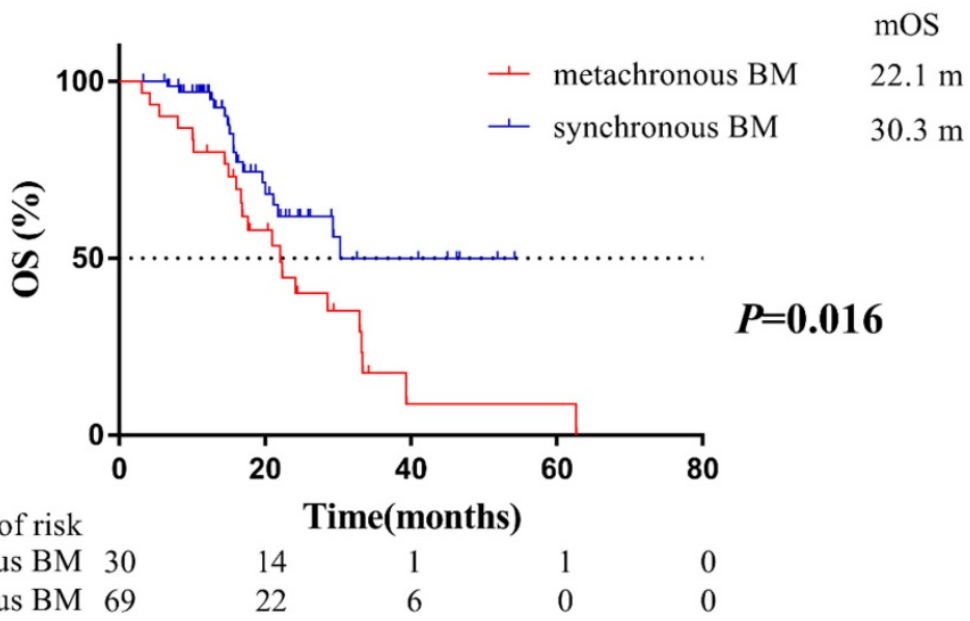

Figure 1. Kaplan-Meier plot of OS in patients with EGFR-mutant advanced NSCLC grouped by BM status. OS, overall survival; NSCLC, non-small cell lung cancer; BM, brainmetastases; $\mathrm{Cl}$, confidence interval.

Table 2. Univariate and multivariate analyses for the factors associated with OS

\begin{tabular}{|c|c|c|c|c|c|c|}
\hline \multirow[t]{2}{*}{ Factors } & \multicolumn{3}{|c|}{$\begin{array}{l}\text { Univariate analysis of OS } \\
(\%)\end{array}$} & \multicolumn{3}{|c|}{$\begin{array}{l}\text { Multivariate analysis of } \\
\text { OS (\%) }\end{array}$} \\
\hline & HR & $95 \% \mathrm{CI}$ & $P$ & HR & $95 \% \mathrm{CI}$ & $P$ \\
\hline \multicolumn{7}{|l|}{ BM status } \\
\hline $\begin{array}{l}\text { Synchronous VS } \\
\text { metachronous }\end{array}$ & 0.462 & $0.243-0.878$ & 0.018 & 0.426 & $0.214-0.846$ & 0.015 \\
\hline Gender: female $V S$ male & 0.801 & $0.420-1.526$ & 0.500 & & & \\
\hline Age, years & 0.972 & $0.942-1.003$ & 0.081 & 0.971 & $0.941-1.003$ & 0.071 \\
\hline $\begin{array}{l}\text { KPS score: } \\
\geq 80 \text { VS }<80\end{array}$ & 0.728 & $0.221-2.390$ & 0.600 & & & \\
\hline BMI & 1.010 & $0.901-1.132$ & 0.865 & & & \\
\hline Smoking: yes $V S$ no & 0.758 & $0.323-1.722$ & 0.508 & & & \\
\hline \multicolumn{7}{|c|}{ Tumor markers level before treatment } \\
\hline CEA $(\mathrm{ng} / \mathrm{ml})$ & 1.000 & $0.999-1.001$ & 0.601 & & & \\
\hline $\mathrm{CA} 125(\mathrm{ng} / \mathrm{ml})$ & 1.000 & $0.999-1.001$ & 0.775 & & & \\
\hline NSE (ng/ml) & 1.014 & $0.988-1.041$ & 0.297 & & & \\
\hline \multicolumn{7}{|l|}{ First-line treatment regimen } \\
\hline $\begin{array}{l}\text { Chemotherapy VS } \\
\text { EGFR-TKIs }\end{array}$ & 1.389 & $0.672-2.869$ & 0.375 & & & \\
\hline Type of EGFR mutations & & & 0.440 & & & \\
\hline 19-del VS L858R & 1.545 & $0.748-3.194$ & 0.240 & & & \\
\hline Other VS L858R & 1.724 & $0.586-5.073$ & 0.323 & & & \\
\hline Type of EGFR-TKIs & & & 0.268 & & & \\
\hline Erlotinib VS Gefitinib & 0.614 & $0.215-1.753$ & 0.362 & & & \\
\hline Icotinib VS Gefitinib & 0.456 & $0.158-1.317$ & 0.147 & & & \\
\hline \multicolumn{7}{|c|}{ NO. of extracranial metastasis } \\
\hline $0-2$ VS 3 or more & 0.523 & $0.181-1.514$ & 0.232 & & & \\
\hline \multicolumn{7}{|c|}{ Location of extracranial metastasis } \\
\hline Pleural effusion & 1.655 & $0.870-3.148$ & 0.125 & & & \\
\hline Liver & 0.779 & $0.186-3.255$ & 0.732 & & & \\
\hline Adrenal & 1.097 & $0.337-3.578$ & 0.877 & & & \\
\hline Bone & 2.027 & $1.062-3.868$ & 0.032 & 1.659 & $0.822-3.350$ & 0.158 \\
\hline $\begin{array}{l}\text { Retroperitoneal lymph } \\
\text { node }\end{array}$ & 2.305 & $0.888-5.984$ & 0.086 & 1.354 & $0.493-3.720$ & 0.556 \\
\hline Other & 1.793 & $0.544-5.916$ & 0.337 & & & \\
\hline $\begin{array}{l}\text { Symptomatic BM } \\
\text { yes } V S \text { no }\end{array}$ & 0.523 & $0.259-1.057$ & 0.071 & 1.294 & $0.537-3.116$ & 0.565 \\
\hline Local therapy for BM & 0.478 & $0.244-0.935$ & 0.031 & 0.357 & $0.159-0.805$ & 0.013 \\
\hline $\begin{array}{l}\text { Radiotherapy for primary } \\
\text { lesion }\end{array}$ & 1.741 & $0.906-3.345$ & 0.096 & 1.437 & $0.665-3.105$ & 0.356 \\
\hline $\begin{array}{l}\text { Subsequent treatment of } \\
\text { Osimertinib }\end{array}$ & 0.442 & $0.213-0.918$ & 0.029 & 0.337 & $0.151-0.751$ & 0.008 \\
\hline $\begin{array}{l}\text { Diagnosed as } \\
\text { oligometastasis }\end{array}$ & 0.531 & $0.222-1.273$ & 0.156 & & & \\
\hline
\end{tabular}

\section{Univariate and Multivariate analysis of OS}

Then 99 patients were further analyzed to explore the prognostic factors associated with OS by univariable and multivariable Cox regression analyses, which were shown in Table 2 . In univariate Cox regression analyses, BM status, bone metastasis, local therapy for $\mathrm{BM}$, subsequent treatment of Osimertinib significantly influenced OS $(\mathrm{P}<0.05)$ (Table 2). Other factors such as gender, KPS score, tumor markers levels before treatment, the first-line treatment regimen, type of EGFR mutations, number of metastases, and diagnosed as oligometastasis were not found to be statistically significant $(\mathrm{P}>0.1)$ (Table 2).

Multivariable Cox regression analysis simultaneously included those factors that had shown associations $(P<0.100)$ in the univariable Cox regression analyses. Moreover, results of multivariable Cox regression analyses showed that $\mathrm{BM}$ status $(P=0.015)$, local therapy for $\mathrm{BM}(P=0.013)$ and subsequent treatment of Osimertinib $(P=0.008)$ had significant impact on OS (Table 2).

\section{Progression-free survival of patients grouped by $B M$ status}

For PFS, a total of 76 patients progressed during follow-up time, whereas 40 patients first progressed in intracranial disease. Among them, this consisted of 20 patients with synchronous BM $(20 / 69,29.0 \%)$, and 20 of patients with metachronous BM (20/30, 66.7\%). The median PFS of these 99 patients was 11.5 months (95\% CI: 8.7-14.2 months). Compared with patients with synchronous BM, patients developing metachronous BM during the course of EGFR-TKIs treatment were at higher risk on PFS $(\mathrm{HR}=1.67$, 95\%CI:1.05-2.66). In detail, the median PFS of 69 patients with synchronous $\mathrm{BM}$ and 30 patients with 
metachronous BM was 12.6 months and 9.4 months, respectively (Figure 2A).

For extracranial PFS, 73 patients progressed during follow-up time. The median extracranial PFS of 69 patients with synchronous BM and 30 patients with metachronous BM was 13.0 months and 14.2 months, respectively. However, there was no significant difference between the two groups $(P$ $=0.56)$ (Figure 2B).

\section{Intracranial progression-free survival of patients grouped by BM status}

For intracranial PFS, a total of 70 patients progressed during follow-up time. The median intracranial PFS of 69 patients with synchronous BM and 30 patients with metachronous BM was 18.7 months and 10.8 months, respectively. It was indicated that patients developing metachronous BM during the course of EGFR-TKIs treatment were at higher risk on iPFS than synchronous BM $(P=0.008$, Figure 3A).

Additionally, there were 71 patients received brain radiotherapy (BRT). Among them, 30 patients received WBRT and 19 patients received stereotactic radiosurgery (SRS) of 69 patients with synchronous $\mathrm{BM}$, and 14 patients received WBRT and 8 patients received SRS of 30 patients with metachronous BM (Table 1). The median intracranial PFS after BRT of 69 patients with synchronous BM and 30 patients with metachronous BM was 18.7 months and 5.4 months, respectively. Our findings also confirmed that patients developing metachronous BM during EGFR-TKIs treatment had poorer responsiveness to BRT than patients with synchronous BM $(P<0.001$, Figure 3B).
$\mathbf{A}$

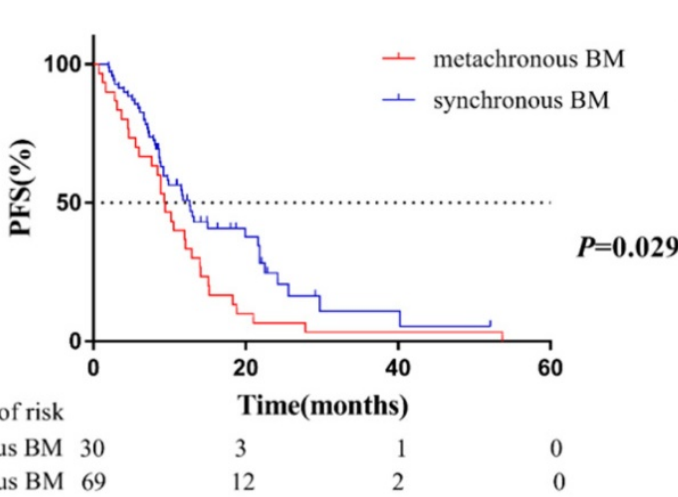

B

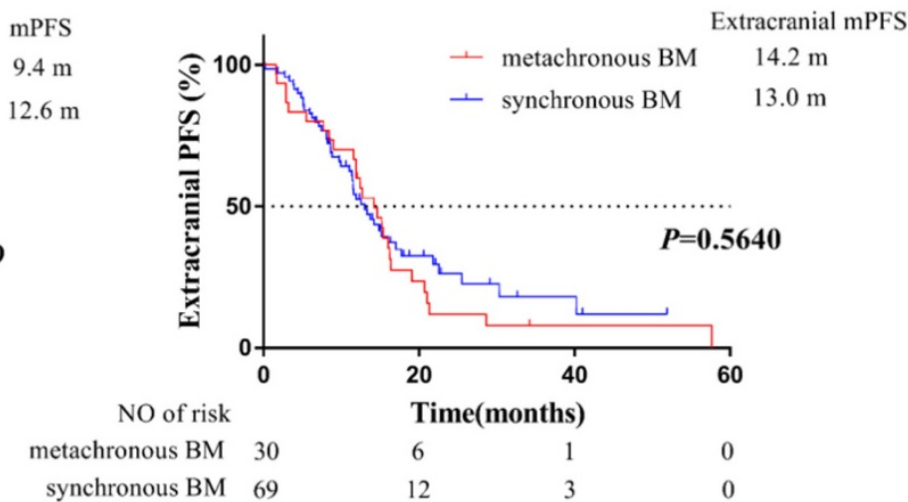

(A) and extracranial PFS (B) in EGFR-mutant advanced NSCLC patients grouped by BM status. PFS, progression-free survival, the time from the EGFR-TKI treatment to PD or death; extracranial PFS, the time from EGFR-TKIs treatment to extracranial PD (exclude intracranial PD) or death from any cause; NSCLC, non-small cell lung cancer; $\mathrm{BM}$, brain-metastases; $\mathrm{Cl}$, confidence interval.

A

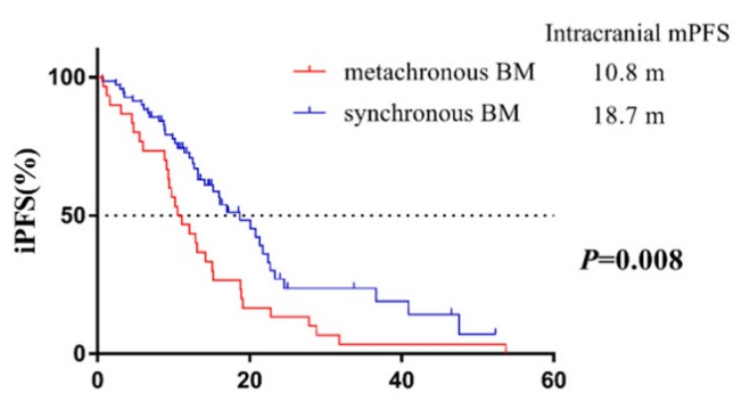

B

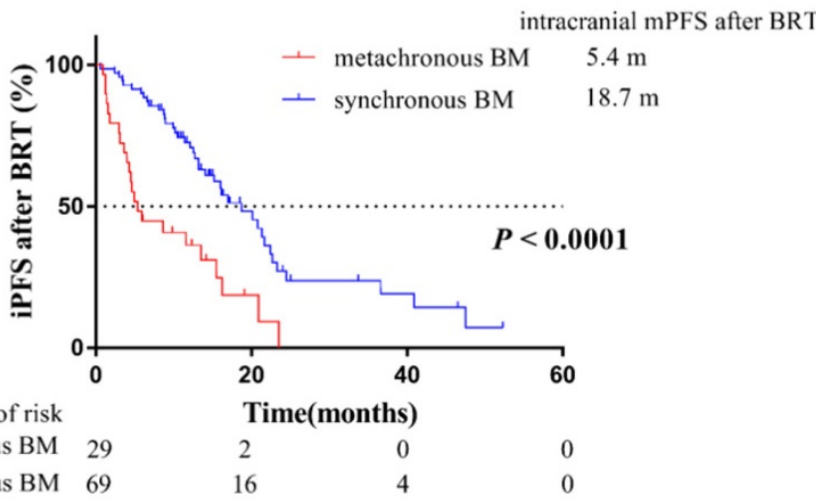

Figure 3. Kaplan-Meier plot of iPFS (A) and iPFS after BRT (B) in EGFR-mutant advanced NSCLC patients grouped by BM status. iPFS, intracranial PFS, the time from EGFR-TKIs treatment to intracranial PD (including appearance of new lesions, or existing lesions progression) or death from any cause; BRT, brain radiotherapy; iPFS after BRT, the time from radiotherapy for intracranial lesions (71 patients who received BRT) or from EGFR-TKIs treatment (28 patients who didn't receive BRT) to intracranial PD or death from any cause; NSCLC, non-small cell lung cancer; BM, brain-metastases; Cl, confidence interval. 


\section{Discussion and Conclusion}

During the past two decades, the advances of EGFR-TKIs revolutionarily improved the prognosis of EGFR-mutant advanced NSCLC patients. The clinical trials of the first- or second-generation EGFR-TKIs showed a median OS of 19.3-33.2 months [14,15]. The prognosis of patients with BM has been considered uniformly poorer than patients without BM [3]. Our results of 99 EGFR-mutant advanced NSCLC patients with BM (including synchronous and metachronous) indicated a median OS of 29.3 months. The OS of patients with BM in our study is roughly similar to the overall patients set including non-BM subset in the first-generation EGFR-TKIs clinical trials [14]. It might be attributed largely to the subsequent usage of third-generation EGFR-TKIs for patients with acquired T790M mutation (Table 1).

Although EGFR-TKIs was reported to be more effective for BM than chemotherapy in EGFR-mutant NSCLC patients [14], there remain some patients developing metachronous $\mathrm{BM}$ during the course of EGFR-TKIs therapy. Lee et al found that $26 \%$ of the patients developed central nervous system (CNS) failure among 166 patients with a clinical benefit to first-generation EGFR-TKIs (gefitinib or erlotinib) treatment [16]. Moreover, compared with NSCLC patients with wild type EGFR, EGFR-mutant patients with longer survival exposed to BM [8], thus patients with EGFR mutation were associated with higher incidence of developing metachronous BM [17]. Therefore, it was suggested more attention should be paid for metachronous BM of patients with EGFR mutant NSCLC whereas there is a lack of sufficient data. To evaluate the impact of BM status on OS, the 99 patients were grouped into synchronous BM and metachronous BM. Our findings first confirmed that patients developing metachronous BM during EGFR-TKIs treatment had poorer outcomes than patients with synchronous $\mathrm{BM}$ at initial diagnosis $(P=$ 0.016, Figure 1).

However, it was also reported that clinical and therapeutic characteristics such as KPS score, local therapy for BM, radiotherapy for primary lesion [18], subsequent treatment of Osimertinib [19], and diagnosed as oligometastasis [20] could influence OS. To eliminate the impact of potential confounders, and to further confirm metachronous BM was an independent prognostic factor of poorer OS, we performed multivariable Cox regression analysis to investigate the potential prognostic factors. Results of multivariable Cox regression analysis indicated that BM status $(P=0.015)$, local therapy for BM $(P=0.013)$ and subsequent treatment of Osimertinib $(P=0.008)$ significantly impacted on OS (Table 2). Significantly, the proportion of local therapy for BM (either WBRT or SRS) had no difference between patients with synchronous and metachronous BM (Table 1). Despite patients with metachronous BM harbored a more favorable prognostic factor (higher proportion of subsequent treatment of Osimertinib, Table 1), but also harbored a poorer prognostic factor, which was metachronous BM. As a result, our results ultimately confirmed that patients that would develop metachronous BM might harbor a more favorable prognostic factor but still indicated a poorer OS than patients with synchronous BM (Figure 1), which confirmed that BM status was the most significant prognostic factor of OS.

Furthermore, we evaluated the PFS of patients grouped by BM status. Our results showed that the median PFS of the overall 99 patients was 11.5 months, which was consistent with the PFS of the first-generation EGFR-TKIs clinical trials [21]. Patients with metachronous $\mathrm{BM}$ were also at higher risk on PFS (HR=1.67, 95\% CI: 1.05-2.66) (Figure 2A). However, for extracranial PFS, our results showed there was no significant difference between the two groups $(P=0.56)$ (Figure 2B), which was suggested that the responsiveness of extracranial lesions to EGFR-TKIs was similar between the two groups. In addition, compared with synchronous BM, higher proportion of patients with metachronous BM (20/30, $66.7 \%$ ) first progressed in intracranial disease. Therefore, our results confirmed that patients of metachronous BM group tended to have a higher risk of intracranial disease progression. It was known that the brain radiotherapy could improve blood-brain barrier (BBB) permeability of EGFR-TKIs. Therefore, the earlier intervene of radiotherapy for $\mathrm{BM}$ in synchronous BM group might partly explained worse outcomes in metachronous BM group.

Our results of median intracranial PFS also confirmed patients of metachronous BM group have a higher risk of intracranial disease progression. In detail, patients developing metachronous BM during EGFR-TKIs treatment had a shorter median iPFS of 10.8 months than patients with synchronous BM of 18.7 months $(P=0.008$, Figure 3A). Then the median iPFS after BRT of 69 patients with synchronous BM and 30 patients with metachronous $\mathrm{BM}$ is 18.7 months and 5.4 months, respectively $(P<0.001$, Figure 3B). It was further confirmed that metachronous BM occurred during EGFR-TKIs treatment had a worse responsiveness to BRT, compared with synchronous BM. The poorer iPFS after BRT partly resulted in a poorer OS of patients with metachronous BM. Therefore, for EGFR-mutant advanced NSCLC, our findings suggested that the patients without $\mathrm{BM}$ at initial diagnosis but would develop metachronous 
BM during the course of treatment harbored a worse intracranial responsiveness to EGFR-TKIs, and those patients should receive more aggressive treatments. And we could recommend patients with high risk of developing metachronous $\mathrm{BM}$ to receive the first-line Osimertinib treatment [13] or prophylactic cranial irradiation (PCI) [22].

In conclusion, the findings of this study are as follows. First, our studies firstly confirmed EGFRmutant advanced NSCLC patients with metachronous BM had worse outcomes than synchronous BM. Second, the multivariate Cox analysis confirmed that BM status was an independent prognostic factor of OS. Third, our results of extracranial and intracranial PFS confirmed that patients of metachronous BM group tended to have a higher risk of intracranial disease progression. Consequently, our findings suggested that the patients without $\mathrm{BM}$ at initial diagnosis but developing metachronous BM during the course of EGFR-TKIs treatment should receive more aggressive treatments. Certainly, there are several limitations in our study that included a retrospective study in a single institution, which inevitably resulted in a selection bias. More finely devised prospective and random study is needed to validate the conclusion.

\section{Acknowledgments}

\section{Funding}

This study was supported by Health Commission of Hubei Province Medical Leading Talent Project and National Natural Science Foundation of China (81502308 and 81773236).

\section{Ethics approval and consent to participate}

This retrospective study was approved by the Ethics Committee of Zhongnan hospital of Wuhan University. Ethics Committee approved oral informed consent, as the data were reviewed and analyzed anonymously. Informed consent was obtained orally from the included patients by telephone.

\section{Consent for publication}

This article is distributed under the terms of the Creative Commons Attribution License which permits any use, distribution, and reproduction in any medium, provided the original author(s) and the source are credited.

\section{Availability of data and materials}

The datasets used and analyzed during the current study are available from the corresponding author upon reasonable request.

\section{Author Contributions}

- Conception and design of the work: Wen Ouyang, Conghua Xie;

- Acquisition, analysis and interpretation of data: Wen Ouyang, Jing Yu, Yan Zhou, Yu Xu, Jie Li, Jun Gong, Junhong Zhang;

- Drafting the work and revising it: Wen Ouyang, Jing Yu, Conghua Xi;

- Final approval of the final version and agreement to be accountable for all aspects of the work: all authors.

\section{Competing Interests}

The authors have declared that no competing interest exists.

\section{References}

1. Siegel RL, Miller KD, Jemal A, et al. Cancer Statistics, 2020. CA Cancer J Clin. 2020; 70 (1):7-30.

2. Kepka L, Cieslak E, Bujko K, et al. () Results of the whole-brain radiotherapy for patients with brain metastases from lung cancer: The RTOG RPA Intra-Classes Analysis. Acta Oncol. 2005; 44 (4): 389-398.

3. Langer CJ, Mehta MP. Current Management of Brain Metastases, with a focus on Systemic Options. J Clin Oncol, 2005; 23 (25): 6207-2619.

4. Lin NU, Wefel JS, Lee EQ, et al. Challenges Relating to Solid Tumour Brain Metastases in Clinical Trials, Part 2: Neurocognitive, Neurological, and quality-of-life outcomes. A Report from the RANO Group. Lancet Oncol. 2013; 14 (10): e407-e416.

5. Maemondo M, Inoue A, Kobayashi K, et al. Gefitinib or Chemotherapy for Non-small-cell Lung Cancer with Mutated EGFR. N Engl J Med. 2010; 362 (25): 2380-2388.

6. Mok TS, Wu YL, Ahn MJ, et al. Osimertinib or Platinum-Pemetrexed in EGFR T790M-positive Lung Cancer. N Engl J Med. 2017; 376 (7): 629-640.

7. Soria JC, Ohe Y, Vansteenkiste $\mathrm{J}$, et a. Osimertinib in untreated EGFR-mutated advanced Non-small-cell Lung Cancer. N Engl J Med. 2018; 378 (2):113-125.

8. Han G, Bi J, Tan W, et al. A retrospective analysis in patients with EGFRmutant lung adenocarcinoma: is EGFR mutation associated with a higher incidence of brain metastasis? Oncotarget. 2016; 7(35): 56998-57010.

9. Bhatt VR, Souza SP, Smith LM, et al. Epidermal Growth Factor Receptor Mutational Status and Brain Metastases in Non-Small-Cell Lung Cancer. J Glob Oncol. 2016; 3 (3): 208-217.

10. Fan $\mathrm{Y}, \mathrm{Xu} X, \mathrm{Xie} \mathrm{C}$. EGFR-TKI therapy for patients with brain metastases from non-small-cell lung cancer: a pooled analysis of published data. Onco Targets Ther. 2014; 7: 2075-2084.

11. Kelly WJ, Shah NJ, Subramaniam DS. Management of Brain Metastases in Epidermal Growth Factor Receptor Mutant Non-Small-Cell Lung Cancer. Front Oncol. 2018; 8: 208.

12. Proto C, Imbimbo M, Gallucci R, et al. Epidermal Growth Factor Receptor Tyrosine Kinase Inhibitors for the Treatment of Central Nervous System Metastases From Non-Small Cell Lung Cancer: The Present and the Future. Transl Lung Cancer Res. 2016; 5 (6): 563-578.

13. Reungwetwattana $\mathrm{T}$, Nakagawa $\mathrm{K}, \mathrm{Cho} \mathrm{BC}$, et al. CNS response to Osimertinib versus Standard Epidermal Growth Factor Receptor Tyrosine Kinase Inhibitors in Patients with untreated EGFR-mutated advanced Non-Small-Cell Lung Cancer. J Clin Oncol. 2018; JCO2018783118.

14. Roeper J, Griesinger F. Epidermal growth factor receptor tyrosine kinase inhibitors in advanced non-small cell lung cancer: what is the preferred first-line therapy? Curr Opin Oncol. 2009; 31 (1): 1-7.

15. Sebastian M, Niederle $N$, Thomas $M$, et al. Molecular genetic tests in advanced non-small cell lung cancer: practical relevance. Dtsch Med Wochenschr. 2014; 139 (41): 2096-2100.

16. Lee YJ, Choi HJ, Kim SK, et al. Frequent central nervous system failure after clinical benefit with epidermal growth factor receptor tyrosine kinase inhibitors in Korean patients with non-small-cell lung cancer. Cancer. 2010; 116 (5):1336-1343. 
17. Hsu F, Caluwe AD, Anderson D, et al. EGFR Mutation status on Brain Metastases from Non-Small Cell Lung Cancer. Lung Cancer. 2016; 96:101-107.

18. Xu Q, Zhou F, Liu H, et al. Consolidative Local Ablative Therapy improves the Survival of Patients with Synchronous Oligometastatic NSCLC Harboring EGFR Activating Mutation Treated With First-Line EGFR-TKIs. J Thorac Oncol. 2018; 13 (9):1383-1392.

19. Ouyang $\mathrm{W}, \mathrm{Yu}$ J, Huang $\mathrm{Z}$, et al. Risk factors of acquired T790M mutation in patients with epidermal growth factor receptor-mutated advanced non-small cell lung cancer. J Cancer. 2020; 11(8): 2060-2067.

20. Ouyang $\mathrm{W}, \mathrm{Yu}$ J, Nuerjiang $\mathrm{S}$, et al. Stereotactic body radiotherapy improves the survival of patients with oligometastatic non-small cell lung cancer. Cancer Med. 2019; 8 (10): 4605-4614.

21. Tetsuya M, Morita S, Yatabe $Y$, et al. Gefitinib versus cisplatin plus docetaxel in patients with non-small-cell lung cancer harbouring mutations of the epidermal growth factor receptor (WJTOG3405): an open label, randomised phase 3 trial. Lancet Oncol. 2010; 11 (2):121-128.

22. Ouyang $\mathrm{W}, \mathrm{Yu}$ J, Zhou $\mathrm{Y}$, et al. Risk factors of metachronous brain metastasis in patients with EGFR-mutated advanced non-small cell lung cancer. BMC Cancer. 2020; 20(1):699. 